\title{
El hecho CIENTífico desde la traducción PARA SU COGNICIÓN
}

\author{
Luz Mery Espinoza VéLez ${ }^{1}$
}

\section{Restumen}

El objetivo de este trabajo es abordar el problema de la traducción, concebida como un proceso de interacción, donde los actores son capaces de transformaciones, asociaciones y conmutaciones que requieren de una estructura de interacción propia de las comunicaciones científicas. Específicamente, analizaré la traducción desde el inglés, en el tratamiento de la ciencia y la tecnología, por ser de interés para investigadores que trabajan en el contexto de la filosofía y de las lenguas con fines científicos y tecnológicos.

\section{Palabras clave}

Traducción, Transferencia, Transustanciación, Intérprete, Interpretante.

\section{Abstract}

The objective of this work is to approach the problem of the translation conceived as an interaction process where the actors are able to transformations, associations and commutations that they require of an interaction structure characteristic of the scientific communications. Specifically, I will analyze the translation from English, in the treatment of the science and the technology, for being of interest for investigators that work in the context of

\footnotetext{
Licenciada en el área de Idiomas de la Universidad Pontificia Bolivariana. Máster en Estudios Avanzados - DEA- Universidad del País Vasco. Candidata al doctorado en Estudios de Ciencia y Tecnología de la Universidad del País Vasco. Decana del Programa de Idiomas del Instituto Tecnológico Metropolitano.

luzespinoza@itm.edu.co
} 
the philosophy and the languages with scientific and technological aims.

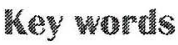

Translation, Transference, Transubstantiation, Interpreter, Interpretant. 


\section{INTRODUCCIÓN}

El inglés de la ciencia y la tecnología implica una doble naturaleza: la actividad académica y la actividad profesional, si consideramos que gran parte de la actividad académica del inglés para fines científicos y tecnológicos se realiza en escuelas de ingeniería, medicina entre otras. Aunque en la actualidad, el inglés de la ciencia y la tecnología es sólo una de las ramas posibles con fines específicos, que ahora se conoce como inglés profesional y académico. (Alcaraz, 2000), además el inglés de la ciencia y de la tecnología es de gran interés en otras modalidades posibles como el inglés de los negocios o el inglés jurídico.

Entre algunos estudios sobre el lenguaje de la ciencia y la tecnología, podemos mencionar la obra ya clásica de Kinneavy (1971). En este trabajo, Kinneavy estudia el lenguaje científico con un interés esencialmente lingüístico y lo define como la variedad utilizada por los especialistas de una disciplina determinada para la transmisión de conocimientos. En concreto, se trata de usar el lenguaje únicamente con el fin de presentar el saber; en el momento que la intención es otra, el lenguaje deja de ser científico (Kinneavy 1971, 88):

Scientific discourse consists in a consideration of one facet of an object and the making of certain kinds of assertions (descriptive, narrative, classificatory, and evaluative) about this facet. A discourse which becomes noticeably expressive or directly persuasive or literarily preoccupied is a discourse in danger of becoming nonscientific.

El lenguaje científico para este autor entonces, es aquél que pretende describir la realidad de forma objetiva.

Por esta razón quizá, Swales titula su libro Writing scientific english (Swales, 1971), cuando en realidad podrá haberse denominado technical english, ya que es un buen ejemplo de una aplicación didáctica que emplea el término inglés científico, para referirse al resultado de cuatro años de enseñanza del inglés en una facultad 
de Ingeniería (Swales ${ }^{2}$, 1985: 38). Swales preparaba a sus alumnos para superar sus estudios de ingeniería y este objetivo es el que también tienen muchos de los trabajos que se publican bajo la denominación de technical writing.

Sin embargo, es evidente el adjetivo científico está ligado al mundo de la investigación y la adquisición del conocimiento, pero los conocimientos codificados e interpretados desde el inglés ,como lengua extranjera, están asociados a los conceptos de la ontología, como una expresión de los conceptos compartidos en procesos de inferencia y transferencia. Es este el significado que desde la filosofía, reconozco y defiendo para el término traducción. La transferencia es la construcción de ontologías, en los procesos de representaciones y organizaciones de los conceptos (humano) sobre el mundo como objeto externo (o no humano).

Desde este punto de vista, y desde la teoría combinatoria de las representaciones científicas propuesta por Andoni Ibarra y Thomas Mormann, planteo como tesis central en este texto: la funcionalidad de la traducción como transformación en un proceso cognitivo de representación homológica y/o homomórfica, con base en los siguientes cinco apoyos teóricos:

- La traducción es inherente al hombre porque él constantemente busca crear signos para representar el mundo.

- La traducción del hecho científico es un puente entre dos mundos: el humano y el no-humano

- La traducción no es una copia simple o reflejo de la realidad, interviene la propiedad del lenguaje de crear y recrear el sentido.

- La traducción es parte de un proceso cognitivo que requiere de la representación del mundo interno para comprender el externo.

- La traducción requiere de un proceso metódico implícito para llegar de A a B y viceversa.

2 Swales 1985: 38. 
En consecuencia, mi propósito es reflexionar sobre las implicaciones de la traducción del hecho científico desde el mundo exterior a éste, especialmente desde el académico de educación superior, desde el cual se leería que el punto de partida $\left(L_{1}\right)$ es el hecho científico, y que el punto de llegada $\left(\mathrm{L}_{2}\right)$ es el concepto elaborado a partir de la lectura hecha sobre el hecho científico. Trataré en la primera parte de reflexionar sobre el tipo de representación, homomórfica y/o homológica que se presenta en la traducción para la cognición del hecho científico. Luego, en la segunda parte, por relación con la primera, intentaré especificar el tipo de traducción que se da en la comprensión del hecho científico.

\section{La maducción desde la teoria combinatoria de las TEPRESENTACIONES WOMOMÓPFICAS Y HOMOLOCLCAS}

¿La traducción acoge parcial o totalmente las percepciones y signos que representan la realidad? En la búsqueda de una respuesta razonable (persuasiva) a esta pregunta, todas las generaciones intelectuales parten de la hipótesis de que hay una realidad fundamental oculta bajo las apariencias, que da cuenta de éstas y que puede ser expresada en alguna medida por medio de símbolos codificados.

La representación en la traducción, a la que me refiero en este artículo, es la representación en la cual la transferencia de información sólo tiene lugar mediante una múltiple serie de transformaciones, no tratándose de una reducción en la traducción, sino de una transustanciación por extensión, definida la transustanciación por Latour" como un cambio de estado tan radical que ahora hay un signo, donde antes había una cosa"3. Es un proceso de conversión, donde se concibe la traducción como un proceso iterativo sobre el referente y la reordenación de representaciones, pero además, de perspectiva constructivista de la ciencia y la tecnología, conocida

3 LATOUR, B. La esperanza de Pandora: ensayos sobre la realidad de los estudios de la ciencia. Barcelona: Gedissa. 2001. p. 81. 
como el enfoque del Actor-red que implica una transformación importante, en el abandono de la idea tradicional de correspondencia literal, término a término, para llegar a la aceptación de un enfoque coherente basado en tres principios heurísticos y retóricos: la simetría ampliada, la heterogeneidad y la ordenación.

Para una mejor comprensión del enfoque del Actor-red (Latour 2001) en los procesos de transformación que se presentan, es conveniente realizar una breve descripción de cada uno de los principios heurísticos mencionados. Primero, por simetría se entiende la consideración de los dualismos habituales en una red de comunicación para producir estabilización y continuidad entre lo verdadero y lo falso, y lo humano y no humano. Segundo, la heterogeneidad en la traducción se fundamenta en su carácter de transversalidad en el tratamiento de tópicos, aspectos y objetos de diversa naturaleza, para los cuales encuentra una referencia. Tercero, la ordenación es un principio en la traducción porque esta misma es una ordenación: afirma la contingencia y el carácter construido a partir de la constitución de lo real como un orden de sus componentes y de los atributos nuevos que maneja para sus conceptos. Estos tres principios favorecen, $\mathrm{u}$ obscurecen, el éxito de la traducción, en tanto que ésta depende de la estabilización y continuidad de los componentes duales de un concepto, la transversalidad del eje temático entre los tópicos del contexto y de la adaptación entre lo previo $\left(\mathrm{L}_{1}\right)$ y lo nuevo $\left(\mathrm{L}_{2}\right)$.

Los estudiosos de la ciencia, en lugar de oponer el mundo y las palabras, han multiplicado los términos intermedios, propiamente las transformaciones y multiplicidad de representaciones generadas en la traducción, entendida ésta, como el intermediario para la solución de oposiciones rígidas entre el contexto y el contenido científico, según la relación e intercambio entre las comunidades científicas. Además la traducción se entiende como el soporte del trabajo de los actores en la modificación, desplazamiento y traslado de sus distintos y contrapuestos intereses. Una mayor optimización del intercambio con el medio crea una tendencia hacia una nueva producción de una correspondencia pragmática entre 
estímulo, representación y respuesta que, en contextos cognitivos polémicos, se presenta como una correspondencia epistemológica. En la concepción de la traducción como transustanciación se considera "que una cosa puede seguir siendo más duradera y puede transportarse más lejos y más rápidamente si continúa sufriendo transformaciones en cada etapa de esta larga cascada" 4 . En este sentido de Latour (2001) se puede leer que la transustanciación es una compresión de la esencia, no sólo por reducción sino también por extensión.

La transformación que ocurre en la traducción es una inscripción de la materialización de una entidad en un signo. Una inscripción o representación tiene las siguientes dualidades: de bidimensión-multidimensión, de asociación-sustitución, de historicidad-transformación, de movilidad-inmutabilidad. Estas características duales de la traducción se manifiestan en la capacidad de trasladar un elemento de un contexto a otro, la transformación de un material para que encaje en un modo de ordenación diferente al del que procede. En suma, la construcción de una nueva disposición, requiere una nueva simplificación acorde con sus nuevas relaciones: ésta es la transformación.

Las dualidades de las inscripciones, son objeto de interpretación y reinterpretación que imprimen, entonces, a la traducción, su fundamento científico representacional porque parten de la representación para la elaboración del nuevo signo. Lo anterior sin desconocer que la traducción y la representación son dos caras de una misma moneda, y son elementos en cuya existencia uno depende del otro. En consecuencia, desde estas dualidades, en la representación a partir de un referente, es posible estudiar la traducción desde la teoría reflexiva combinatoria de las representaciones.

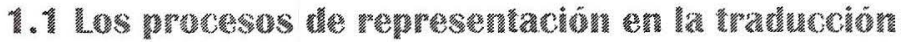

De manera facilista se podría hablar de la traducción de una representación sobre el hecho científico. En un acto de esa natura-

Ibid. p. 74 . 
leza no se tendría en cuenta que la representación en un proceso cognitivo es un acto interno sobre el cual todavía la psicología cognitiva no tiene la última palabra. Se trata de un acto humano. Sin embargo, no sin precaución y de manera a priori, es posible pensar que la expresión de la representación se puede lograr por una traducción elaborada desde el mismo sujeto, en el contacto de los dos extremos del hecho científico: humano-no humano.

La asociación y la substitución como funciones intelectuales propias de la representación permiten la elaboración de nuevos conceptos. Por ello la relación en pareja de estos dos mecanismos sustituye a la obsoleta distinción entre sujeto-objeto. Entonces, cuando se trabaja sobre la comprensión de un hecho científico, la traducción, como una conversión, proporciona un conjunto de símbolos (palabras) elaborados a través de asociaciones y substituciones en el escenario de la representación. Considerada la traducción desde la perspectiva facilitadora, gracias a la asociación y a la substitución de las propiedades de los conceptos, es preciso tener en cuenta que su acción es funcional en torno al contexto, del cual dependen los tipos de asociación y de sustitución, pero conservando el referente. Por ejemplo, desde la filosofía de la historia, que no sólo se refiere al paso del tiempo, sino también al hecho de que algo ocurre en el tiempo, la dualidad historicidad y transformación hace que la traducción no se refiera solamente a ese algo (los hechos) que simplemente pasa, sino algo que genera transformación, algo no sólo compuesto por fechas, sino por acontecimientos (hábitos de acción de Peirce); no integrado sólo por intermediarios, sino también por mediaciones en contextos distintos. Es la relación de los hechos la que conserva o no, las estructuras y organizaciones de los objetos empíricos.

Si la asociación y la sustitución son dos funciones de la representación y ésta a su vez determina la traducción, en nuestro caso de un hecho o concepto científico, cabe la pregunta sobre su incidencia en los tipos de representación homomórfica y homológica. Desde el concepto de "referencia circulante" de Latour (2001), además relacionado con el de la distribución de la ciencia, la asociación 
y la sustitución permiten articular proposiciones. Por lo tanto la traducción no es un equivalente término-término; se da en virtud del análisis que ofrece claridad sobre el referente.

En concordancia con lo anterior, una representación homomórfica en un proceso de traducción se caracteriza por el análisis según la estructura de la relación del concepto $A\left(L_{1}\right)$ y el concepto $B\left(L_{2}\right)$, en cuyo caso la estructura del concepto $B$ es representante de la de A, si se da la adaptación de A en B. Porque es la estructura una manera de conservar el referente. Ahora, aunque se habla de una representación por estructura, se da también una por lógica $u$ homológica, ya que se logra una relación lógica por estructura. Y es esa relación lógica la que permite oxigenar el concepto de una cultura a otra, e incluso la apropiación de la forma de nominar conceptos, nombrándolos con los mismos sonidos de otro idioma. Así la estructura de un concepto, al interior de la ciencia, depende de las prácticas realizadas sobre el referente.

Ilustrando la estructura de la relación entre los conceptos A y B, donde A se concibe a partir de un referente lingüístico, se observa que cuando se adiciona sal a los alimentos, por costumbre, tradición o sabor, se hace referencia a la salubridad. Desde el concepto de la cotidianidad: salado, no salado o más o menos salado, y quizás se guarde en su forma de uso la prevención con respecto a la salud, ante la experiencia o no sobre las incidencias nocivas de estos elementos para el organismo, si se utilizan en forma excesiva. Desde el trabajo académico, se conocen las características del $\mathrm{NaCl}$ y sus componentes: el cloro y el sodio; ya las referencias posibles son las reacciones químicas, la proporción de cantidad de cada elemento, o entre muchas otras el peso y la masa. Desde lo epistemológico, corresponde saber cuáles son los modelos utilizados para intervenix las ciencias que se han encargado de estudiar la sal.

En la acción académica el agente, dígase el estudiante o el estudioso, se enfrenta a la abstracción de los conceptos del hecho científico o derivados de éste, como el de $\mathrm{NaCl}$. El acercamiento se da porque no puede aprehenderse la ciencia sin los datos. Ahora bien, estos datos, que interrelacionados informan acerca del hecho 
científico, no deberían ser un fin en sí mismos, sino que deben ser un medio para acceder a otras formas de información y comprensión, en lo posible de mayor avance y complejidad. Los datos no se justifican si no promueven conductas o conocimientos significativos que lleven a la construcción de los conceptos por la interrelación de datos nuevos con el conocimiento previo y con otros conceptos.

Desde las funciones intelectuales de la representación, se presenta la conservación de la estructura del concepto y la movilidad. Las transformaciones como móviles-inmutables se ubican en el espacio entre el sujeto y el objeto, además obligan por naturaleza a que en la acción del pensamiento se busque la traducción de ese espacio externo que la mente trata de representar y concretar mediante proposiciones. En los posibles desplazamientos, similitudes y contradicciones presentadas en el proceso de concatenación de los elementos de todos los signos, presentes a la hora de concretar una representación, la referencia circulante entre todos los signos hace posible el móvil inmutable que trabaja Latour y que bien se podría denominar también oxigenador del referente. Es decir, si en la traducción se presentan transformaciones, éstas se dan gracias a que ellas mismas son móviles-inmutables. Aunque de todos modos se genera la pregunta iprimero es la transformación y luego la traducción? Según lo antes expuesto, el proceso es un todo integrado, muy posiblemente la traducción también genera transformación en el mismo instante del proceso de desplazamiento. Lo cierto del caso es el puente que se extiende entre los actores de los dos mundos: el interno (la ciencia) y el externo (el mundo político), así este último dependa de las relaciones de poder.

El carácter de movilidad e inmutabilidad es lo que facilita la comprensión frente a la reducción, y en lo que consiste la referencia circulante. Se presenta entonces el carácter homológico, de manera tal, que se convierte en el instrumento para des-cubrir a través de la lógica, el mundo ya dado, pero oculto (cubierto). Cuanto más estructurado está un medio social más suelen estarlo sus sistemas de codificación-decodificación y menos problemáticas suelen ser sus representaciones. La mayoría de éstas se hacen por 
analogía, "siguiendo la regla" de casos previos. No obstante, dado que ninguna regla prescribe exhaustivamente qué cuenta como su cumplimiento, nunca se puede dar una representación por definitiva. Esto hace conveniente interesar a otros elementos en la red de la representación (en especial, prácticas de asociar). La simetría ampliada propone que ha de usarse el mismo tipo de explicación para todos los elementos de la misma red, sean aparatos, grupos sociales o fuerzas naturales. La primera implicación de este principio es considerar todos los dualismos habituales como productos de un trabajo de movilización de materialidades heterogéneas en red para producir un efecto de estabilización o dicotomía-que puede, por otra parte, construirse de nuevo en una continuidad-.

La dualidad de bidimensión y multidimensión promueve más un homomorfismo y una carencia de isomorfía. Para desenvolverse en el flujo de lo real, todas las culturas utilizan prácticas cognitivas que diferencian emisores/receptores de signos y signos que remiten arbitraria y convencional pero discerniblemente a ciertos objetos. La utilidad pragmática de estas operaciones da lugar a la diferenciación contextual y provisional de "humanos", "no humanos" y "signos". Esta distinción tripartita es una primera catalogación básica de lo real que distingue contextualmente entre: lo natural (que habla sin palabras) y lo simbólico (que emplea palabras y otros signos) La cuestión es cómo se genera la distinción entre mundo y observador, entre signo y agente simbolizador, en suma, entre actor y actante, en cuya relación también existe una transustanciación.

\section{2 g}

Mi reflexión sobre la traducción y su apertura comunicativa y transformadora se basa en fundamentos cognitivos del concepto científico, teniendo en cuenta que las unidades de conocimiento van más allá de la apropiación del término, es decir, se sitúan en una operación cognitiva multirelacional, en donde el mundo se construye y se desconstruye, se forma y se transforma, se estabiliza y se desestabiliza a través de una construcción lógica realizada 
por los actores individuales y colectivos, humanos y no humanos. Estos tienen la necesidad de traducir sus problemas, identidades y lenguajes en los de otros.

En estas cadenas de traducción se presentan diferentes actividades: estrategias concurrentes, confrontaciones, movilizaciones, inscripciones, alianzas, asociaciones y desplazamientos, con la consecuencia en las transformaciones de los esquemas: reproducción por cambio, micro y macro. Según estas actividades se presentan otras cadenas (procesos) de traducción: reducción, compresión, marcado, continuidad, reversibilidad, estandarización y compatibilidad.

Es preciso notar la presentación de procesos diferentes, mientras en la producción científica se busca la correspondencia entre las palabras a través de una compresión (se busca un signo por cuatro palabras, ejemplo: eutanasia: muerte sin sufrimiento físico), en la actividad humana se da un proceso de cognición, se busca un equivalente que mantenga el sentido del referente enviado por la ciencia, (se buscan cuatro palabras para un signo, ejemplo: exploración de las cavidades internas del organismo: endoscopia). En esta última no hay compresión, sino extensión: no es una reducción por traspaso de sentido, sino una extensión por transustanciación. ¿Cuándo se llega de A a B por R homológica dónde está la traducción?. La traducción exige un proceso metódico de transformación realizada para llegar de A a $B$ y viceversa. La traducción, con respecto a la abstracción, es el puente que pernite el transporte y las transformaciones y transmutaciones posibles manteniendo la referencia. Pero, en la serie de transformaciones se siguen unas pautas para poner en relación distintos marcos de referencia, espacios, tiempos y personajes.

Según como se realice esta serie de transformaciones del plano de referencias, es preciso considerar tres etapas. Estas son: Una paso a: cuando el intérprete es enviado de un plano de referencia a otro. La vuelta a: cuando el intérprete es remitido nuevamente al plano de referencia original. Cambio a: cuando el contenido de la expresión varía por completo. Estas tres etapas contribuyen a 
la producción de un referente interno y a la vez proporcionan diferentes transformaciones. En estas transformaciones se transita entre dos o más mundos, se enriquece a la ida y a la venida y se está constantemente explorando a profundidad las novedades lingüísticas, se conocen los valores culturales de un mundo (dígase, por ejemplo, de un concepto), que están incrustados en sus discursos, entonces, el intérprete ofrece una ventana a la otra cultura, a través de su trabajo logra transmitir los valores culturales y fomentar el intercambio verdadero de ideas dando lugar a la formación de nuevas culturas.

La traducción entendida desde la lingüística, puede ser un acto exterior, pero me refiero al acto de traducción que a su vez conlleva el proceso de traducción como transferencia. Es entonces, un proceso de transustanciación. Si tenemos en cuenta el significado de la palabra sustancia, que se refiere a aquellas propiedades que subyacen, en las traducciones se conservan las propiedades que caracterizan el significado dado al referente, pero se transmutan las transformaciones aportadas en los procesos de representación. De esta manera se logra la circulación del referente, gracias al "llevar" o "ducción", de la referencia. De esta afirmación era consciente Carnap (1988), y sostenía que en la ciencia las propiedades se determinan por la disciplina y su método, sin embargo, en los humanos la asignación de propiedades se da de forma natural, por emoción, sensación y percepción. Pero en los procesos de traducción como en los de un espacio académico, desde Latour "esa palabra sustituye a una cosa, mientras conserve una característica que la defina" ${ }^{2}$.

El sentido se mantiene (paso a, vuelta a y cambio a), gracias a esas características, pero según el tipo de representación que haya sido elaborada, porque esas propiedades pueden corresponder a la lógica (representación homológica) o a la estructura (representación homomórfica) o a una combinación de las dos. La traducción comparte con la lógica el interés fundamental por los conceptos,

$5 \quad$ Ibid, p. 80 . 
principalmente la relación que se establece entre el objeto de la realidad y el concepto que lo representa. De la existencia de los distintos objetos de la realidad, el individuo pasa al concepto o a la clase de objetos por un proceso de abstracción. Para llevar a cabo este proceso, se prescinde de las características contingentes e irrelevantes de los objetos individuales y se retiene simplemente las que son pertinentes para la caracterización de la clase que representa la diversidad.

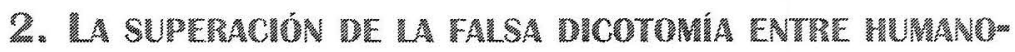

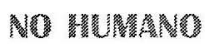

Ante la dicotomía tradicional objeto-sujeto, señalo aquí en primer lugar la categoría humano no humano, frente a la pretensión de la perspectiva analítica de los científicos su fundamento ontológico y el contexto donde surgen sus teorías. En segundo lugar, explicito ambos conceptos intentando profundizar en sus potencialidades de representación, para luego hacer referencia a las principales implicaciones epistemológicas y metodológicas en el abordaje de estudios empíricos.

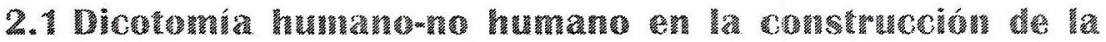 Ciñ}

El objetivo en este trabajo es asumir la reflexión sobre la caracterización de lo humano y lo no humano, desde la pretensión de tomar consciencia sobre algunos de los aspectos de la realidad humana: el Actor-Actante con sus funciones y metas, en la actividad de la comprensión sobre la práctica de la ciencia.

Tal actitud metodológica lleva consigo la convicción de que la mera descripción de las condiciones objetivas no logra explicar totalmente el condicionamiento social de las prácticas. En este trabajo, entonces, se rescata al actor social, no en cuanto individuo, sino como agente socializado que interviene el hecho científico en el momento de abordar su abstracción. Para lograr este objetivo es necesario romper con la falsa dicotomía planteada ya en el naci- 
miento de las ciencias sociales por el objetivismo y el subjetivismo y sustituir la relación ingenua entre el individuo y la sociedad, por la relación construida entre los dos modos de existencia de lo social: las estructuras sociales externas y las estructuras sociales internalizadas. La necesidad de superar esa disyuntiva se fundamenta en una especial afirmación de Latour “...de la simetría del Actor-actante nos fuerzan a abandonar la dicotomía sujeto-objeto, una distinción que impide la comprensión de los colectivos" ${ }^{\text {. }}$

Para explicar la simetría entre humanos y no humanos es preciso recurrir a lo que se conserva a través de las transformaciones, es decir, el conjunto de competencias y de propiedades de intercambio entre los actores y actantes. De esta manera, los científicos no son grandes pensadores dedicados a la cuestión de confirmar o refutar teorías, sino también hombres ligados a la resolución de problemas concretos de investigación. Estos hombres también pueden ser no conscientes de los supuestos teóricos de su actividad. De esta manera, prácticamente se aborda a Kuhn desde su noción de "paradigma", en tanto que ayuda a desmitificar la noción de lo que son los científicos, su actividad y sus productos.

Es evidente que se presentan obstáculos inconmensurables de comunicación en las comunidades lingüísticas. Siendo uno de esos problemas la traducción. Kuhn propone un análisis a esos problemas de traducción, cuya labor califica de "ardua y que a menudo resulta en equívocos que pueden conducir a desacuerdo". Uno de los problemas identificados en la traducción (manifiesta de diferentes formas en la comunicación de los conocimientos), es la conexión del referente con el contexto cultural, en el espacio interno o externo de la ciencia; se continúa el estudio de Gadamer ${ }^{7}$ al considerar que el conocimiento es fundamental para la existencia humana, y que la persona sólo desde su propio horizonte de interpretación, que se construye constantemente, puede comprenderse y comprender

$6 \quad$ Op. cit., p. 216.

7 GADAMER, Hans-Gerg. Verdad y método, Ediciones Sígueme: Salamanca, 1991 pp. 38-48. 
su contexto. Igualmente, Habermas ${ }^{8}$, con el concepto de acción comunicativa, introduce una noción complementaria: el mundo de la vida, único horizonte desde el cual y sobre el cual puede producirse la reproducción simbólico-social en acciones lingüísticamente mediadas.

Siempre entre la ciencia y la sociedad ha existido la mediación del científico con todas sus debilidades y fortalezas, su medio, sus intereses y sus acciones como entidades con propiedades asociadas. Pero, la mayoría de los científicos cree habérselas directamente con la realidad, no asumen estar mediados por supuestos conceptuales específicos. El científico - en consonancia con lo anterior, y en contra de posiciones como la de Popper- no es un desinteresado buscador de verdades, sino un sujeto socialmente condicionado que busca, en primer lugar, legitimarse dentro de la comunidad científica. El elemento objetivo de su posición no es la referencia a una realidad incontaminada, sino a una situación social objetiva dentro de un campo de relaciones de poder en el aparato institucional de los científicos, donde no se busca el conocimiento abstracto de utilidad para la ciencia, sino un concreto reconocimiento. Sus decisiones, algunas veces están afectadas por situaciones contextuales ajenas a lo científico mismo, de las cuales a menudo el científico, puede no ser consciente.

\subsection{Implicaciones epistemológicas y metodolögicas an ell intento de superar la dicotomía sujeto-objeto}

En la construcción teórica, fundada en una epistemología bachelardiana, en la cual "el hecho científico se conquista, se construye, se comprueba", se da cuenta de las implicaciones del significado de lo no humano enmarcado en el ámbito existente entre humano-no humano, superando la dicotomía sujeto-objeto, propiamente con referencia a las potencialidades inscritas en los cuerpos de los agentes y en la estructura de las situaciones donde actúan, o más

HABERMAS, Jürgen. Teoría de la acción comunicativa. Racionalidad de la acción y racionalización social. Vol. I Madrid: Taurus. Humanidades, 278. 1987a. 
exactamente en su relación no entendida como objetos conocidos por un sujeto, ni objetos manipulados por un amo, ni ellos mismos amos de nada, sino que por humano- no humano se responde a la inquietud contemporánea cuando se exige que lo social fuera explicado por lo social y sólo por lo social. Esta posición pretende explicar, entonces, las prácticas sociales -hasta dónde ello es posible-desde una perspectiva sociológica y como si fueran totalmente explicables sociológicamente. Así, un humano es el agente que se constituye como organismo, sólo si dispone de un sistema especializado y autónomo de procesamiento de información (un centro) que regula íntegramente su conjunto, es decir, que actúa con otros materiales y la realidad en una interacción, proceso; la vida: comunicación, intercambio.

El humano en la práctica de las ciencias, se relaciona con otros humanos y con no-humanos, para ello, dispone de múltiples sistemas simbólicos de comunicación, incluyendo el lenguaje verbal -hablado y escrito-, los gestos, los sistemas de medida, las imágenes, entre otros. Las percepciones son mensajes de retroalimentación de la interacción con el medio, la recepción de mensajes simbólicos lo es específicamente de interacciones lingüísticas con sus semejantes.

Para actuar con la realidad, el humano y las culturas utilizan prácticas cognitivas que diferencian emisores/receptores de signos y signos que remiten a la utilización pragmática de ciertas operaciones mentales como la interpretación y la representación que ayudan a la diferenciación contextual y provisional de "sujetos", "objetos" y "signos". Esta distinción tripartita es una primera catalogación básica de lo real que distingue contextualmente, entre lo natural (que habla sin palabras), lo social -como individualidad socializada-(que emplea palabras) y lo ritual -como colectividad articulada o unidad/relación con la trascendencia- (donde reside la eficacia del significado). De esta manera el humano emplea la traducción como elemento de importancia en la producción y distribución de los resultados de las prácticas científicas y por lo tanto del conocimiento científico. Por esta razón procederé a reflexionar sobre la 
traducción como un proceso de representación y transformación con relación a la referencia u objeto de la ciencia.

\section{CONCLUSIONES}

- En este análisis sobre la representación en la traducción, ésta se concibe como un proceso de interacción donde los actores son capaces de transformar, asociar, conmutar y guiar fuerzas y entidades que requieren de una estructura de mediación con los fenómenos, de manera que puedan traducirse en alguna forma de inscripción, propias de las comunicaciones científicas.

- Gracias a los trabajos de traducción como transformación y como representación de representaciones, se puede rastrear y cartografiar la migración del conocimiento y la cultura en los principales centros del conocimiento.

- El papel del traductor o intérprete es de importancia en la transferencia de tecnología del mundo desarrollado al mundo en desarrollo. La traducción logra transmitir la cultura y fomentar el intercambio de valores, contribuyendo a que las fronteras se vuelven menos definidas y se transita, entonces, por zonas más híbridas y fluidas.

- En la distribución del conocimiento en la educación, el traductor de manera didáctica desde diversas áreas científicas y técnicas tiene que entender y dilucidar al texto para luego ponerlo al alcance del lego. Es decir implica investigación, representación $\mathrm{y}$ transformación para producir y distribuir el conocimiento.

- El carácter de movilidad e inmutabilidad es lo que facilita la comprensión frente a la reducción, y en lo que consiste la referencia circulante. Se presenta entonces el carácter homológico, de manera tal, que se convierte en el instrumento para des-cubrir a través de la lógica, el mundo ya dado, pero oculto (cubierto). Cuanto más estructurado está un medio social más suelen estarlo sus sistemas de codificación-decodificación y menos problemáticas suelen ser sus representaciones. 
- El mundo se construye y se desconstruye, se forma y se transforma, se estabiliza y se desestabiliza a través de una construcción lógica realizada por los actores individuales y colectivos, humanos y no humanos. Estos tienen la necesidad de traducir sus problemas, identidades y lenguajes en los de otros. En estas cadenas de traducción se presentan diferentes actividades: estrategias concurrentes, confrontaciones, movilizaciones, inscripciones, alianzas, asociaciones y desplazamientos, presentándose las transformaciones de los esquemas: reproducción por cambio y micro por macro. Según estas actividades se presentan otras cadenas (procesos) de traducción: reducción, compresión, marcado, continuidad, reversibilidad, estandarización y compatibilidad. 


\section{BibLografia}

ALCARAZ, E. El inglés profesional y académico. Madrid: Alianza. 2000.

CARNAP, R. La construcción lógica del mundo. México, UNAM, 1988.

IBARRA, Andoni. MORMAN, Thomas. Representaciones en la ciencia. De la invariancia estructural a la significatividad pragmática. Barcelona: Ediciones del Bronce. 1997.

(Una teoría combinatoria de las representaciones científicas. En: Crítica, Revista Hispanoamericana de Filosofia. Vol. XXXII - No. 95México, agosto, 2000. 3-45.

GADAMER, Hans-Gerg. Verdad y método. Salamanca: Sígueme, 1991, pp. $38-48$.

HABERMAS, Jürgen. Teoría de la acción comunicativa. Racionalidad de la acción y racionalización social. Vol. I Madrid: Taurus. Humanidades, 278. 1987a.

KUHN, T. The structure of Scientific Revolutions. Chicago: University of Chicago Press, 1970.

LATOUR, B. La esperanza de Pandora: ensayos sobre la realidad de los estudios de la ciencia. Barcelona: Editorial Gedissa, 2001.

MOSCOVICI, Serge. On social representation. En: J. P. Forgas (Compilador). Social cognition. Perspectives in everyday life. Londres: Academic Press, 1981.

The phenomenon of social representations. En R.M. Farr y S. MOSCOVICI (Comps.). Social representations. Cambridge: University Press, 1984.

PEIRCE, Charles S. Philosophical writings of Peirce. The principles of Phenomenology, 1940.

Escritos lógicos. Introducción, selección y traducción de Pilar Castrillo Criado. Madrid: Alianza, 1968.

SWALES, J. Genre. Analysis. English in Academic and Research Settings. Cambridge: University Press, 1990. 